\title{
LA PERSPECTIVE FONCTIONNELLE DE LA PHRASE DANS UNE PERSPECTIVE HISTORICO-PHILOLOGIQUE DU FOYER PRAGOIS DE STRUCTURALISME FONCTIONNEL
}

\author{
Tomáš HosKOVEC \\ Université Masaryk, Brno
}

\begin{abstract}
En): The "functional sentence perspective" and the "topic-focus articulation" (of a sentence) designate now two different schools within the Prague linguistic tradition. Yet to Vilém Ma-the-sius, the founder and promoter of the Prague structuralist programme, and the pioneer of functional re-search into sentence meaning, they rather represented two terminological variants. To him, a perspective was a way of bringing together sen-tences differing in their formal grammatical shape, but similar in their textual (utterance) function, so that their respective meaning differences could be described by detailed mutual oppositions. The "functional", i.e. communicative perspective, articulating about what (topic) something is conveyed (focus) when the sentence is uttered, is only one perspective among others Mathesius took into account. The article explains why, in the classical period of the Prague Linguistic Circle, sentence phonology was consciously kept apart from the study of communicative sentence perspective, and outlines how, in the post-classical periods, that branch of studies ramified into different schools.
\end{abstract}

Keywords (En): Prague Linguistic Circle, Vilém Mathesius, Prague centre of functional structuralism, functional lin-guistics, sentence meaning, sentence perspective, topic-focus articulation.

Mots-clés (Fr): Cercle de Prague, Vilém Mathesius, foyer pragois de structuralisme fonctionnel, linguistique fonc-tion-nel-le, signification de la phrase, perspective de la phrase, articulation actuelle, thème et rhème.

La perspective fonctionnelle de la phrase, sous ses appellations les plus diverses, est un sujet «pragois » autant typique et presque autant notoire que la phonologie, mais de renom différent. Tandis que la phonologie est considérée même par bon nombre de linguistes - affaire close, réglée et claire, la perspective fonctionnelle de la phrase a la réputation d'être restée inachevée, confuse, obscure. Peu de philologues le disent ouvertement (K. J. DOVER, 1960), beaucoup le pensent et avouent en privé (maintes communications personnelles), la majorité en «votent à pied sec » en contournant le problème. Nous sommes persuadé que les deux renoms sont également faux : la phonologie est loin d'être achevée (ALBANO LEONI, 2009), rien que pour la description de la phrase, la perspective fonctionnelle de la phrase a beaucoup à nous apprendre, rien que, méthodologiquement, pour le rapport entre le système abstrait de langue et les textes ou discours concrets. En outre, se pencher sur les recherches des perspectives (sic !) de la phrase dans le courant du structuralisme fonctionnel du foyer pragois est une excellente occasion de se repérer dans la complexité historique, et notamment philologique de la linguistique actuelle.

Tout comme il n'existe pas une seule phonologie pragoise, il n'existe pas non plus une seule théorie pragoise de perspective fonctionnelle. Il est fascinant d'observer les bouleversements de l'actualité que la question a connus : elle est 
posée et réglée sans faire écho bien avant la création même du Cercle linguistique de Prague; elle est rappelée lors des premières présentations internationales de celui-ci pour être presque immédiatement repoussée comme un ensemble de phénomènes dont on reconnaît l'importance, mais que l'on préfère tenir à l'écart afin de mieux s'occuper d'autres choses; elle réapparaît formulée comme une tâche majeure dans les dernières années du premier Cercle, sous l'occupation nazie, pour renaître avec une vigueur inattendue dès la première stabilisation (post-)stalinienne du pays communiste, lorsque le Cercle ne peut plus, depuis longtemps, exercer ses activités; elle a l'attraction d'un fruit défendu sous l'immobilité brežnevienne, pour se trouver abandonnée sous la liberté recouvrée, au profit d'activités diverses; à l'heure actuelle et dans son «pays d'origine », elle semble désuète, aux yeux des générations nouvelles, et imprégnée d'un certain esprit de chapelle. Force est alors d'y remédier !

1. Dans l'histoire intellectuelle du foyer pragois de structuralisme fonctionnel, la notion de perspective «fonctionnelle » de la phrase, que l'on appelle parfois aussi «communicative », est étroitement liée avec le personnage de Vilém Mathesius, fondateur et, jusqu'à sa mort, survenue en 1945, président du Cercle linguistique de Prague. En racontant le chemin intellectuel du Cercle, on commence d'habitude par la conférence publique Sur la potentialité des phénomènes linguistiques faite par Mathesius en 1911 (MATHESIUS, 1911) à la Société Royale des Sciences de Bohême (cf. MATHESIUS, 1936b ; VACHEK, 1999 : 43). Or à ce moment-là, Vilém Mathesius avait déjà publié, presque en même temps que sa thèse de doctorat La critique de Shakespeare par Hippolyte Taine (1907-1908), sa thèse d' «habilitation » (terme à interpréter à la suisse) intitulée Études sur l'évolution de l'ordre des mots en anglais (1907-1909). C'est cette dernière qui nous servira de point de départ.

Le choix du sujet était peu commun à l'époque, et l'approche adoptée l'était encore moins, mais nous savons de ses Mémoires que MATHESIUS (2009 : 209201), encore étudiant, s'était choisi lui-même - pour deux de ses trois mémoires de fin d'études - des analyses de l'ordre des mots dans deux vieux textes, un tchèque et un allemand, et qu'il était fier d'avoir réussi à faire accepter son choix à ses professeurs. La perspective fonctionnelle de la phrase n'était pas pour Mathesius une découverte faite au bout de longues recherches, c'était une évidence qu'il voyait d'emblée comme un des phénomènes en fonction desquels le sujet parlant, lors de la création de son texte (discours), choisit et arrange les moyens d'expression de la langue. L'évidence de cette fonction était telle que Mathesius ne songeait même pas à lui donner une existence terminologique nette : il la décrivait comme il lui convenait.

MATHESIUS (1924) ne revient à ce propos qu' au bout de quinze ans, toujours avant la création du Cercle, survenue en 1926. Il parle alors - en tchèque - du rapport entre gramatické členění věty 'articulation grammaticale de la phrase' et rozdělení větného obsahu na thema a vlastní výpověd' 'division du contenu de la phrase en thème et énonciation propre'. Il en reparle en anglais (MATHESIUS, 1928) en terme de «two different conceptions of the grammatical subject, that of the doer of the action expressed by the predicative verb and that of the theme of 
the enunciation contained in the predicate », à quoi il ajoute la note : «By the terms 'theme' and 'enunciation' I mean what is usually called the psychological subject and psychological predicate, respectively. » Voilà la clé d'interprétation pour la partie $2 b$ des Thèses du Cercle linguistique de Prague (TCLP, 1: 13) où l'on distingue, en termes assez aléatoires, entre «la division formelle [de la phrase] en sujet et prédicat grammaticaux » et «la division actuelle de la proposition en thème et énonciation». D'ailleurs, tout le premier paragraphe de ce passage des Thèses reproduit l'argumentation de MATHESIUS (1924, 1928).

$\mathrm{Si}$ à cette époque-là Mathesius cherche à introduire quelque chose dans la terminologie scientifique, c'est la «caractérologie linguistique » dont il parle, en anglais en terme de linguistic characterology, au Premier congrès international de linguistes à La Haye en 1928 :

The distinguishing feature of linguistic characterology is the introduction into linguistic analysis of the conception of value and synchronic interrelations. If it is the task of the descriptive grammar to give a complete inventory of all formal and functional elements existing in a given language at a given stage of its development, linguistic characterology deals only with the important and fundamental features of a given language at a given point of time, analyses them on the basis of general linguistics, and tries to ascertain relations between them. (MATHESIUS, $1928 \equiv 1930: 56$ )

...et récrit en allemand en 1929 en terme de linguistische Charakteristik. La caractérologie souhaitée de Mathesius n'a malheureusement pas pris racine dans la linguistique moderne, ${ }^{1}$ mais le terme de Satzperspektive qu'il a mis spontanément dans le titre de son article allemand (MATHESIUS, 1929a) a fait fortune trente ans plus tard : Jan FIRBAS (1957) s'en est servi pour éviter la traduction malcommode de «division actuelle de la phrase », utilisée à l'occasion dans les Thèses (TCLP, $1: 13$ ), en donnant naissance à Perspective fonctionnelle de la phrase, Funktionale Satzperspektive, Functional sentence perspective; nous y reviendrons ultérieurement au paragraphe 4.

2. Peu après sa réapparition aux premiers débuts du Cercle linguistique de Prague, la perspective fonctionnelle est consciemment mise à l'écart. Le Cercle de Prague, depuis 1931 représenté au CIPL (Comité international permanent de linguistes) et chargé de la direction de l'AIEP (Association internationale pour les études phonologiques), se concentre sur la phonologie. Si à l'époque nous rencontrons des phénomènes liés à la perspective fonctionnelle de la phrase, ils sont mentionnés de façon négative. Ainsi Roman JAKOBSON (1931), analysant la nature de l'accent, commence par séparer strictement l'accent du mot (phonotactique) de l'accent de la phrase (syntagmatique). Ce faisant, il donne un bel exemple d'opposition de perspective fonctionnelle de la phrase, qu'il récuse aussitôt comme étant sans intérêt :

\footnotetext{
${ }^{1}$ Dommage qu'il en ait été ainsi : le concept des valeurs résultant des rapports mutuels au sein d'un système, tel qu'il est contenu dans la caractérologie de Mathesius, est structuraliste nec plus ultra.
} 
Die Abstufung der Syntagmabetonungen (die Satzbetonung) dient zur Differenzierung der Satzbedeutungen. Ein čechisches Beispiel: “dones 'Janovi 'tuto 'knihu - bringe Jan dieses Buch, tu es doch ; 'dones "Janovi 'tuto 'knihu - es ist Jan, dem du das Buch bringen sollst ; 'dones 'Janovi "tuto 'knihu — es ist dieses Buch, das du Jan bringen sollst ; 'dones 'Janovi 'tuto "knihu - bringe Jan dieses Buch und nicht irgendein anderes Ding. (JAKOBSON, 1931: 165 $1962: 118)$

Dans une note de bas de page, Jakobson précise que son exemple est pris d'un manuel du tchèque pour les lycées tchèques et ajoute qu'une monographie contemporaine allemande sur l'accent mentionne, cette fois-ci pour l'allemand, «des farces bien connues » qui consistent à changer la signification d'une phrase rien qu'en déplaçant son accent. Il faut en déduire que les phénomènes de signification qui font pour nous l'objet propre de la perspective fonctionnelle de la phrase étaient connus sans pourtant être pris en compte. Ceci est confirmé par Serge KARCEVSKIJ (1931) qui, en esquissant les fondements d'une phonologie de la phrase, déclare expressément :

La scission de la phrase (nous n'appliquerons ici ce terme que pour désigner la division de la phrase en deux parties) n'a rien à voir avec la distinction d'un sujet et d'un prédicat, ni avec aucune opposition d'entités grammaticales, en général. Nous préférons également éviter les termes si mal déterminés mais si bien compromis de sujet et prédicat logiques. Nous n'en avons d'ailleurs point besoin, puisque c'est la structure formelle et non le contenu psychologique qui intéresse le linguiste.

(KARCEVSKIJ, $1931: 205 \equiv 2000: 103)$

Les deux études que nous avons citées jusqu'ici, JAKOBSON (1931) et KARCEVSKIJ (1931), font partie du volume 4 des Travaux du Cercle linguistique de Prague, Réunion phonologique internationale tenue à Prague (18.-21.XII 1930), et elles y sont les seules à s'occuper, malgré que négativement, de la signification de la phrase. Encore une troisième étude du même volume s'approche du domaine, celle de Jan MUKAŘOVSKÝ (1931), intitulée «La phonologie et la poétique ». Une fois de plus, la pertinence de la prosodie pour la signification de la phrase, c'est-àdire du vers, y est reconnue pour être tout de suite repoussée. Ce à quoi Mukařovský aspire, c'est la totalité des traits acoustiques pertinents pour le sens d'une œuvre poétique. Voyons à ce propos sa conclusion :

L'application du point de vue phonologique est donc d'une utilité essentielle pour l'analyse du côté phonique de l'œuvre d'art littéraire :

$1^{\circ}$ Elle aide à établir une délimitation entre les qualités purement acoustiques (que l'on pourrait aussi appeler «déclamatoire»), facultatives au point de vue de l'œuvre, et entre ces éléments phoniques qui sont des parties intégrantes de sa structure.

$2^{\circ}$ Elle donne la possibilité de s'occuper du rapport structural entre le côté phonique et les autres éléments du poème.

Ce rapport n'est pas celui d'un parallélisme automatique, mais celui d'une interaction mutuelle et active entre les membres des deux groupes. Si l'on ne se rend pas compte de cette interaction, il est sûr que dans toute analyse on déformera l'un et l'autre côtés de l'œuvre. (MUKAŘOVSKÝ, $1931: 287$ )

Rappelons que pour Mukařovský, cet «autre côté », à savoir le « deuxième groupe » (par rapport aux sonorités) comprend toute la structure de l'œuvre 
littéraire telle qu'elle est construite avec les moyens de langage, dont les motifs singuliers qui font les matériaux de l'œuvre, et les thèmes généraux de l'œuvre, qui résultent chacun d'un arrangement particulier de tels matériaux. ${ }^{2}$ Mukařovský avait pour principe de reconnaître d'emblée la pertinence significative de toutes les composantes systémiques de langue (phonèmes, prosodèmes, lexies, syntagmes, tous confondus), et se défendait par principe d'élever une composante quelconque au-dessus des autres.

$\mathrm{Au}$ même temps, Mathesius publie en tchèque, dans deux revues philologiques différentes, deux articles traitant de deux phénomènes qui, dans le système de la langue tchèque, servent à moduler la perspective fonctionnelle, c'est-à-dire communicative, de la phrase. Or tandis que MATHESIUS (1930) explique l'ordre des mots en tchèque parlé à l'aide de la perspective fonctionnelle de la phrase, il observe (1931) la ligne dynamique de la phrase tchèque en toute indépendance d'elle. ${ }^{3}$

Lorsque MATHESIUS (1936b) dresse un bilan bien détaillé des premières dix années du Cercle de Prague, il ne dit pas un mot sur la perspective fonctionnelle. Dans le volume 6 des Travaux du Cercle linguistique de Prague, paru également en 1936 sous le titre Études dédiées au Quatrième congrès de linguistes, le phénomène de perspective fonctionnelle (communicative) de la phrase n'est mentionné qu'une seule fois lorsque Vladimír SKALIČKA (1936) énumère, de façon systématique, les arrangements possibles des unités linguistiques. La même absence peut être constatée, pour l'année 1937, au journal du Cercle Slovo a slovesnost [Le verbe et la création verbale], où pourtant Vilém MATHESIUS (1937a,b) traite de l'intonation de la phrase, aussi bien qu'au volume 8 des Travaux, Études phonologiques dédiées à la mémoire de M. le Prince N. S. Troubetzkoy.

Je comprends cette absence comme voulue : la prosodie de la phrase qui était alors le grand défi porté au Cercle, devait être examinée sans aucun a priori. On voulait se rendre à l'évidence des phénomènes prosodiques immanents, dotés certes de pertinence significative à l'instar des phonèmes, mais aucunement secondaires par rapport à une stratégie langagière quelconque. C'était à l'époque LE problème qui empêchait le Cercle d'avancer dans le domaine qui nous

\footnotetext{
${ }^{2}$ Dès ses débuts, MuKAŘOvSKÝ (1928) s'efforce de concevoir l'œuvre poétique, c'est-à-dire l'œuvre d'art littéraire, en tant que structure autonome faite à partir des moyens de langage, à force de saisir les notions traditionnellement reconnues pour «extralinguistiques » (idées, sentiments, événements, etc.) comme résultant de diverses structurations de divers faits de langage. Or le langage étant une institution socio-culturelle, la production aussi bien que la réception de pareilles structures langagières n'est possible que si elles sont soumises à un ensemble de normes et de valeurs (les deux actes, production et interprétation, s'appuyant sur le même ensemble de normes et de valeurs), reconnues par le collectif devant lequel l'ouvre apparaît. - Voilà une belle contrepartie de la caractérologie de Mathesius (cf. n.1), elle aussi structuraliste par excellence.

${ }^{3}$ La ligne dynamique de MATHEsius (1931) équivaut la ligne expiratoire de Mukařovský, qui, inspiré par l'esthéticien Otokar Zich, distingue trois composantes principales, mais non uniques, de la ligne phonique, à savoir : ligne expiratoire (accroissement et diminution de l'intensité expiratoire, sommets expiratoires, pauses), ligne d'intonation (élévation et abaissement du ton [hauteur] de la voix), mode de liaison des syllabes dans la prononciation (lié, non lié). Parallèlement à MATHESIUS (1931), MUKAŘOVSKÝ (1929) étudie les rapports de la ligne phonique avec l'ordre des mots dans les vers tchèques et touche des phénomènes de la perspective fonctionnelle de la phrase sans jamais les nommer.
} 
concerne ici : on comprenait intimement la perspective fonctionnelle (communicative) de la phrase en tant que stratégie stylistique, choix conscient des moyens systémiques de langue en vue de créer un énoncé concret, et précisément pour cette raison on écartait la perspective fonctionnelle de l'inventaire du système phonologique de langue, même pour les langues, dont le tchèque, dans lesquelles la prosodie sert la perspective fonctionnelle de la phrase à un degré éminent.

3. Rappelé discrètement en 1938 , le phénomène de perspective fonctionnelle de la phrase réapparaît au Cercle de Prague pour la deuxième fois - et de pleine force - en 1939. Deux grands porteurs de la phonologie sont alors absents : Troubetzkoy mort, Jakobson exilé. Mais ceci n'est pas la raison. Le retour en avait été annoncé par une nouvelle vague de discussions sur la nature de la phrase et sur la conception de grammaire structurale, discussions menées par Vilém MATHESIUS (1936a) et Vladimír SKALIČKA $(1935,1937){ }^{4}$

La grammaire structurale fait l'objet d'une inégalité majeure dans la transmission de la connaissance du foyer pragois de période classique : certains membres du Cercle linguistique de Prague de l'époque, qui par la force des choses avaient alors une double affiliation, passent actuellement pour des représentants exclusifs de leur autre foyer (le genevois Serge Karcevski, le strasburgeois Lucien Tesnière), un membre a fait vraiment beaucoup pour incorporer toute l'époque pragoise dans son évolution linguistique individuelle (l'américain Roman Jakobson), plusieurs membres n'ont pas fait grand-chose pour répandre la longue élaboration de leurs idées (les pragois Bohuslav Havránek, Bohumil Trnka, Pavel Trost, Vladimír Skalička et maints autres) ; un autre foyer européen manifestait déjà à l'époque l'intention de reprendre des pragois le flambeau structuraliste pour passer du stade phonologique au stade grammatical (les danois Viggo Brøndal et Louis Hjelmslev, membres eux-aussi du Cercle de Prague). Devant une complexité pareille des faits nous devons nous contenter, à l'heure actuelle, d'une ébauche très simplifiée visant uniquement à faire comprendre Mathesius dans son contexte historique.

Lorsque Vilém Mathesius organisait les premières rencontres de débat avec de jeunes philologues prometteurs, dont Karcevskij, Jakobson, Trnka, Havránek (voilà le noyau du futur Cercle linguistique de Prague !), il était déjà porteur, depuis des années, d'une vision très nette de la linguistique, c'est-à-dire de la philologie fonctionnelle. Sa vision englobait deux pôles, le système abstrait de langue, d'un côté, et les énoncés concrets, de l'autre. Les énoncés de Mathesius, comprenant à la fois les discours oraux et les textes écrits, sont des communications concrètes qu'il faut [savoir] saisir comme des faits sociaux complexes : ils ont toujours un aspect « rationnel » et un aspect «affectif, émotionnel»; un côté « informatif» qui oriente le destinataire vers le monde extra-linguistique (auquel monde extra-linguistique peut appartenir parfois aussi le destinateur lui-même, désirant informer sur son humeur et sur ses intentions), et un côté «esthétique » qui en même temps oriente le destinataire vers l'énoncé même ; ils ont toujours la

\footnotetext{
${ }^{4}$ Pour simplifier les choses, nous passons sous silence le côté slovaque du Cercle de Prague, où de telles questions sont abordés notamment par L’udovít NovÁK $(1936,1937)$.
} 
dimension socio-culturelle qui seule garantit que divers membres d'un même collectif peuvent concorder dans leur interprétation. La langue est alors un inventaire de moyens expressifs appropriés à servir, en synergie choisie, les divers buts que les énoncés concrets peuvent poursuivre. Foncièrement philologue, Mathesius plaide l'équivalence fonctionnelle de la linguistique et de la stylistique, qui ne sont différenciées que par leur orientation: tandis que la linguistique scrute les énoncés concrets afin d'établir les moyens dont dispose la langue du collectif qui les a produits, la stylistique scrute les énoncés concrets afin d'établir comment ils sont faits dans leur individualité à partir des moyens dont dispose la langue du collectif qui les a produits.

Partagée par tout le Cercle de Prague, cette vision de Mathesius était suffisamment porteuse pour inspirer de nouvelles recherches et suffisamment floue pour permettre des approches très diverses. Loin de tout préconçu, la structure de la langue se manifestait comme une tâche à entamer. Mathesius lui-même se limitait aux contours généraux du système de langue. ${ }^{5}$ Le langage, dit-il, s'empare de la réalité en la simplifiant au maximum : de toute la richesse de l'expérience vécue, le langage ne choisit que quelques morceaux qu'il met en quelques relations explicites au détriment de toute une gamme de rapports également existants. Motivé par cette image, qui d'ailleurs était bien proche de la conception de Bally et de Sechehaye (cf. l'auteur $2011: 54$ ), Mathesius postule deux actes concurrents qui créent l'énoncé, la dénomination et la mise-en-relation, tout en soulignant que le premier nommé ne précède le deuxième que lors d'un exposé didactique des choses, tandis que dans le langage même les deux actes agissent simultanément. En conséquence, il faut présupposer, pour le système abstrait de langue, deux domaines principaux : le lexique, inventaire des moyens pour l'acte de dénomination, et les schémas phrastiques, inventaire des moyens pour l'acte de mise-en-relation ; et pour la linguistique en tant que science, deux branches majeures : une onomatologie fonctionnelle et une syntaxe fonctionnelle (le double usage de l'épithète «fonctionnelle» est authentique de Mathesius). Par contre, la morphologie, qui s'imbrique dans les deux actes créateurs d'énoncés, n'est pas une branche particulière de la linguistique ; et chose d'autant plus curieuse, la phonologie, gloire du Cercle de l'époque, reste en dehors de la linguistique fonctionnelle

\footnotetext{
${ }^{5}$ Il est dans les meilleures traditions du structuralisme européen d'osciller entre les termes « structure » et «système». Dans les statuts du Cercle linguistique de Copenhague, forum institutionnel du foyer structuraliste danois, nous lisons : «Lingvistkredsen, der er stiftet d. 24.September 1931 i København, er en Arbejdssammenslutning for Forskning inden for almen og systematisk Sprogvidenskab », littéralement : «Le Cercle de linguistes, institué à Copenhague le 24 septembre 1931, est un groupement de travail pour la recherche dans la linguistique générale et systémique » (c'est nous qui soulignons). Le premier cahier des Acta linguistica, « revue internationale de linguistique structurale », entreprise commune des Cercles de Prague et de Copenhague, abandonnée à cause de la guerre imminente, aux mains des seuls danois, se termine par une voix dissidente de Hjalmar Lindroth, membre du conseil international de la revue, qui souhaite remplacer le terme de «structuralisme » par celui de "systémologie », le structuralisme étant à ses yeux souillé par diverses associations négatives. Or la seule chose qui compte est la vision du rapport entre le tout et les parties : est structuraliste (systémologique) chaque approche qui reconnaît que le tout n'est pas la somme de ses parties, mais l'ensemble des rapports qui unissent les parties.
} 
n'étant qu'une science auxiliaire qui traite des éléments expressifs des moyens linguistiques propres. ${ }^{6}$

Soulignons que pour Mathesius, la phrase est un énoncé élémentaire remplissant certaines conditions, qui ne nous concerneront pas pour l'instant, donc un texte (écrit ou oral) concret par lequel un destinateur s'adresse à un destinataire en adoptant certaines attitudes et en poursuivant certains buts. Si alors la aktuální členění větné ('articulation actuelle de la phrase') est classée, par Mathesius et ses contemporains, fait stylistique, cela ne veut point dire «chose mineure »: la stylistique vaut autant que la linguistique, la différence entre elles ne résidant que dans l'optique, pour ne pas dire dans la «perspective» du chercheur. Au fait, l'étude de l'effet, c'est-à-dire du sens d'une disposition particulière de moyens systémiques de langue dans un texte concret - en l'occurrence, dans une phrase - est stylistique de par sa nature.

Et rappelons en fin de compte, que dès ses débuts, le Cercle linguistique de Prague envisage saisir les concepts littéraires traditionnels de motif, sujet et thème d'un texte comme des faits purement linguistiques. C'était surtout Jan MUKAR̆OVSKÝ $(1928,1938)$ qui s'assignait cette tâche, se penchant exclusivement sur des œuvres poétiques, alors que Vilém MATHESIUS (1941a, 1942, et notamment 1943) s'occupait de textes prosaïques, banalement utilitaires.

4. Regardons maintenant de plus près l'appareil notionnel de Mathesius. Pour éviter toute confusion, suspendons à ce paragraphe 4 l'usage du terme «perspective fonctionnelle de la phrase », qui comme nous avons relevé à la fin du paragraphe 1, ne remonte qu'à Jan FIRBAS (1957), en le remplaçant par celui de perspective «communicative» de la phrase. Cela nous permettra de parler librement d'autres perspectives encore, fonctionnelles elles aussi et concernant inévitablement la phrase.

L'expression de «perspective [communicative] de la phrase» ([kommunikative] Satzperspektive) est tout-à-fait occasionnelle de Mathesius (1929a), qui ne s'en est servi qu'une seule fois écrivant en allemand, tandis qu'en tchèque il ne l'utilise jamais. Sa contrepartie tchèque aktuální členění větné a mis un temps considérable à s'imposer, chez lui, comme entité terminologique : elle manque dans ses articles 1924, 1930, 1931 (sans parler de sa thèse 1907-1909), elle s'annonce dans le 1938 sous forme de aktuální členění obsahu větného vzhledem ke kontextu, ce que Mathesius lui-même (1939a: 411) rend en allemand comme aktuelle Gliederung des Satzinhaltes mit Rücksicht auf den Kontext, pour être introduite dans le titre de l'article 1939b à l'aide de «sur la soi-disante », après quoi elle compte enfin pour terminologiquement fixée (Mathesius 1941a,b, 1942, 1943). Dans son article 1938, qui annonce la stabilisation terminologique finale, Mathesius reprend une tournure occasionnelle utilisée auparavant dans le 1929b où il dit aktuální členění větného obsahu, at’ jde o pořad objektivní nebo

\footnotetext{
${ }^{6}$ Sous un tel jour, la phonologie bénéficie d'un statut pareil à celui de l'orthographe, domaine dans lequel le Cercle de Prague a également introduit une recherche fonctionnelle, remplaçant d'un coup de balai les explications historiques isolées par une nouvelle vision englobante des moyens expressifs du langage écrit (cf. ARTYMOVYČ, 1932a,b; VACHEK, 1939, 1942).
} 
subjektivní 'articulation actuelle du contenu de la phrase, qu'il s'agisse de l'ordre objectif ou subjectif'. Ce fut alors au même temps qu'il écrivit en allemand (1929a) Zur Satzperspektive im modernen Englisch: la «perspective» et l'«articulation » sont donc nées sous la même étoile.

Nous n'avons pas de terme français authentique de Mathesius. La «division actuelle de la phrase » que nous lisons dans les thèses du Cercle de Prague (TCLP $1: 13$ ) équivaut à un autre terme, rozdělení, utilisé lui aussi à l'occasion par Mathesius (1924). En outre, nous devons avouer une mauvaise chance en cherchant le terme français là où il faut, c'est-à-dire dans la revue tchèque du Cercle Slovo $a$ slovesnost, qui dans le contenu sommaire de chaque année, accompagnait régulièrement les titres d'articles et de rubriques de leur traduction en français. Or avec l'occupation nazie du pays, cette agréable coutume a dû céder — proprement à l'année où la terminologie se fixe. Toujours est-il que la traduction est univoque : appuyé sur le parallélisme du tchèque členění $(\leftarrow$ členit $\leftarrow$ člen) et de l'allemand Gliederung $\leftarrow$ (gliedern $\leftarrow$ Glied $)$, on doit mettre articulation $(\leftarrow$ articuler $\leftarrow$ article).

Constatons surtout que pour MATHESIUS (1882-1945), contrairement à l'évolution ultérieure de la linguistique tchèque dans la deuxième moitié du $\mathrm{XX}^{\mathrm{e}}$ siècle, il n'y a pas d'opposition entre «perspective » et «articulation» de la phrase. Il scrute minutieusement la structuration du texte minimal qu'est la phrase en ce qui est énoncé (rhème) à propos de quelque chose (thème) sans pourtant s'arrêter là : l'articulation propre, à savoir la division du contenu de la phrase en deux parties, n'est pas un but à soi. Mathesius s'intéresse toujours à la stratégie globale du texte, aux raisons du choix de l'ordre des parties articulées (thème rhème vs. rhème - thème), aux raisons du choix de ce qui sert de thème, aussi bien que de ce à quoi sert le rhème, parce que, comme nous avons rappelé à la fin du paragraphe 3, il s'efforçait, avec le Cercle tout entier, de concevoir diverses unités du contenu de texte comme des unités linguistiques. C'est précisément cette complexité des fonctions textuelles qui est selon nous LA raison du retard terminologique de la aktuální členění větné dans ses écrits.

Une digression apparente peut s'avérer très instructive ici. En 1940, Mathesius publie un article intitulé L'expression de la perspective passive dans la phrase tchèque dans lequel il introduit une "perspective passive », terme tout neuf et concept d'autant plus neuf, opposée à la voix passive, terme et concept grammaticaux des plus traditionnels possibles. Il ne cache point son ambition de trancher sur le débat invétéré et stérile, si ou non le tchèque (en particulier, et les langues slaves en général) possède(nt) la catégorie de genus passiuum à l'instar du latin et des langues occidentales modernes. ${ }^{7}$ Sa perspective passive est une stratégie langagière, donc communicative, un ensemble de fonctions d'après lesquelles le sujet parlant, c'est-à-dire l'homme de paroles localisé dans un collectif socio-

\footnotetext{
${ }^{7} \mathrm{Au}$ foyer pragois de structuralisme fonctionnel, le problème avait fait l'objet de la monographie de Bohuslav Havránek $(1928,1937)$, récit extraordinaire d'un processus d'acculturation de toute une famille de langues européennes qui, ne disposant à l'aube de leur histoire attestée d'aucune différence morphologique de voix verbale, ont développé des séries entières de structures syntaxiques pour servir de nouvelles fonctions apprises aux étalons culturels grec et latin. Sur la position de ce travail au foyer pragois, cf. l'auteur 2011.
} 
culturel particulier, fait son choix parmi les moyens offerts par le système abstrait de langue afin d'exprimer tel ou tel aspect de l'affectation du sujet textuel momentanée par l'action du prédicat verbal.

Mathesius travaille à la fois avec la sémantique du verbe au prédicat, qui au sein du système abstrait de langue, peut toujours être remplacé par un autre verbe, avec la structure actantielle immanente du verbe choisi, inséparable de la sémantique verbale, avec le fait particulier que le verbe comprend, ou pas, parmi ses actants l'agent de l'action qu'il exprime, avec le besoin éventuel d'évacuer un tel agent, besoin réalisé à l'aide des moyens systémiques de langue. Par exemple, à propos du verbe se tuer, il ne vaut pas grand chose de dire que c'est un passif réflexif par rapport à la fois à tuer et à être tué, parce qu'il est beaucoup plus important de voir que le sujet (grammatical) est impliqué consciemment dans l'action, ce qui permet à se tuer d'exprimer à la fois un suicide et un accident, pourvu que le sujet prenne une partie consciente et active à l'action qui a mené à cet accident : il s'est tué en grimpant au Cervin: : il a succombé aux blessures causées par sa chute: : il a été victime d'une avalanche; il s'est tué en voiture / en avion [qu'il conduisait] : : il a péri lors d'un accident de voiture / d'avion [n'étant pas au volant]; il s'est tué en manipulant maladroitement le fusil : : il a été tué par le mari jaloux [qui de son fusil, a tiré sur lui].

Pour mieux le comprendre, soulignons que Mathesius refuse, certes, une approche que l'on pourrait qualifier d' «onomasiologie grammaticale», à savoir manipulation mécanique des formes systémiques, sans pourtant prêcher une sémasiologie. Son approche, qu'il qualifie de «fonctionnelle», consiste à introduire, dans la linguistique, le souci du choix d'ensembles définitoires appropriés au sein desquels le linguiste peut différencier de façon fructueuse des figures de langue de taille diverse, y compris la taille d'une phrase. A l'époque où l'étude différentielle du signe linguistique binaire peinait encore énormément à se mettre en route, Mathesius, qui ne parle jamais du signe, comprend à merveille comment s'y prendre. ${ }^{8}$

A la fin du paragraphe 3, nous avons dit que pour Mathesius, la phrase était un énoncé élémentaire (donc un texte) remplissant certaines conditions. Lesquelles? Voilà ce qu'il met dans sa définition :

La phrase est un énoncé élémentaire communicatif par lequel le sujet parlant adopte une attitude active vis-à-vis d'une réalité, et qui paraît formellement habituel et subjectivement complet. (MATHESIUS, $1923 \equiv 1947: 231$ )

Ici « communicatif » s'oppose à «affectif, émotionnel » (Mathesius reconnaît que dans chaque énoncé, les deux pôles sont présents: c'est donc la prépondérance du rôle communicatif, non l'absence de l'affectivité qui caractérise la phrase); la forme « habituelle » est celle des schémas syntaxiques offerts par le

\footnotetext{
${ }^{8}$ Sur ce point, Mathesius était secondé par Jan Mukařovský développant l'étude différentielle de signes linguistiques de la taille de tout une œuvre poétique, cf. l'auteur 2012. Les analyses linguisticostylistiques de Mathesius, Mukařovský, Havránek et autres, entreprises lors de la période classique du foyer pragois de structuralisme fonctionnel, méritent bien être considérées des cas de sémantique interprétative avant la lettre.
} 
système de langue (Mathesius parle, en tchèque, de «clichés », son disciple SKALIČKA (1936) utilise le même mot lorsqu'il écrit en français); "subjectivement complète» est la phrase par son intonation (la phrase répugne à l'incomplétude intonatoire).

Mathesius conçoit donc la phrase comme une disposition d'unités lexicales (acte de dénomination, cf. § 3 ) selon des règles de nature morphosyntaxique (acte de mise-en-relation, cf. § 3), le tout étant toujours doté d'un contour intonatoire (pertinent même pour les phrases écrites). Il ne voit aucun intérêt à y identifier des concepts grammaticaux traditionnels, non parce qu'ils sont mal définis (Mathesius est bien conscient des problèmes inhérents des parties du discours d'origine logico-ontologique), mais parce qu'ils ne disent rien sur le contenu sémantique de la phrase. C'est pourquoi il introduit, dans la linguistique, diverses perspectives, divers ensembles de fonctions, lesquelles fonctions, seules et à elles-mêmes, justifient le choix de telle ou telle unité lexicale insérée dans tel ou tel cliché (morho)syntaxique et prononcée sur tel ou tel ton. Les fonctions sont identifiées de façon différentielle : le choix d'une autre unité lexicale, d'un autre cliché morphosyntaxique, ${ }^{9}$ d'un autre contour intonatoire, traduirait une autre vision de la « réalité », une autre «attitude » adoptée vis-à-vis de celle-ci. Revoyons alors à la lumière de tout cela le contexte des études de Mathesius de ses dernières années :

- Dans les 1938 et 1939a, il introduit une double perspective de renforçage et d'emphase (Verstärkung und Emphase) afin d'élucider un phénomène souvant constaté, jamais précisé. Il le définit comme marque d'intensité dans la phrase, et puisque la phrase naît d'un double acte langagier, l'intensité marquée tombe nécessairement soit dans l'acte de dénomination ( $\rightarrow$ renforçage), soit dans celui de mise-en-relation ( $\rightarrow$ emphase). Les fonctions d'un tel marquage peuvent être très diverses : à l'acte de dénomination, le marquage concerne non seulement le degré quantitatif, mais aussi, voire surtout, une évaluation positive ou négative ; à celui de mise-en-relation, le marquage concerne soit l'articulation actuelle du contenu de la phrase compte tenu du contexte (aktuelle Gliederung des Satzinhaltes mit Rücksicht auf den Kontext), soit l'attitude caractéristique vis-à-vis du contenu de la phrase, adoptée par le sujet parlant (charakteristische Einstellung des Sprechenden zum Satzinhalt). L'opposition bien stricte de renforçage et d'emphase selon les deux actes constitutifs de la phrase est une exigence théorique incontournable, mais Mathesius ne tarde pas à concéder que dans chacun des deux, peuvent à l'occasion être empruntés des moyens typiques de l'autre. ${ }^{10}$

\footnotetext{
${ }^{9}$ Nous avons dit plus haut (§ 3) que Mathesius, postulant une syntaxe fonctionnelle à côté d'une onomatologie également fonctionnelle, niait, à la morphologie, le statut de discipline linguistique autonome. Puisque la morphologie s'imbrique foncièrement dans les deux actes constitutifs du langage, à savoir dénomination et mise-en-relation, sur l'opposition desquels Mathesius envisageait bâtir sa nouvelle linguistique théorique, il devait la tenir à l'écart par la force des choses. Cependant, il ne niait aucunement la pertinence de divers procédés morphologiques pour la syntaxe. Et comme les clichés syntaxiques en question ne sauraient même pas être décrits sans recours à des termes morphologiques, nous préférons parler de clichés «morphosyntaxiques ».

${ }^{10}$ Il vaut la peine de relire, dans la perspective unificatrice de renforçage/emphase, divers petits articles rédigés par Mathesius dans les années 20, dont notamment le 1926, où il traite du pronom démonstratif tchèque utilisé souvent sans raison apparante, qui a déjà à mainte reprise suscité la question si ou non nous assistons à la naissance d'un article défini en tchèque.
} 
- Dans le 1940, il présente la perspective passive, que nous avons expliquée ci-haut, alternative fonctionnelle au passif purement formel des grammaires traditionnelles.

- En 1941, il retravaille son ancien concept d'apposition (1923), prédication latérale ajoutée à un membre quelconque de la phrase, en ouvrant ainsi une perspective prédicative qui permet de distinguer la prédication majeure de la phrase d'éventuelles prédications mineures. Ceci permet un traitement un et commun des deux types principaux de clichés phrastiques qu'il appelle «prédicatifs» (phrases à deux membres, dans la terminologie (onto)logicogrammaticale traditionnelle) et «thétiques » (phrases à un seul membre).

- Depuis 1939 jusqu'à 1943, il élabore la perspective communicative, moyen pricipale pour identifier des unités textuelles fondamentales, telles que motif, sujet, thème (cf. ci-haut, le dernier alinéa du paragraphe 3 ).

5. Nous avons dit au début du paragraphe 3 que le débat sur l'articulation acutelle de la phrase reprend au Cercle linguistique de Prague en même temps qu'y naît le premier débat sur la grammaire structurale. C'est une fois de plus Vilém MATHESIUS (1936a) qui a ouvert le débat structural, ayant fait comprendre à Vladimír Skalička le vrai potentiel théorique de sa thèse de doctorat (1935), mais il s'en retire aussitôt, laissant l'affaire aux jeunes du Cercle, à savoir Skalička et Ludovít Novák. ${ }^{11}$ Pour sa personne, Mathesius choisit une solution fonctionnelle, appuyée sur l'analyse des textes. Toutes les perspectives mentionnées ci-haut (§ 4), qu'elles soient ou non appelées par lui ainsi, sont des ensembles de fonctions sémantiques motivant divers assemblages de phrases, au sein desquels les phrases doivent être étudiées différentiellement par opposition mutuelle. Il ne veut plus entendre parler de recherche sur la signification d'un phénomène linguistique singulier : aussi s'oppose-t-il farouchement à František TRÁVNÍČEK (1937, 1939), membre vénéré du Cercle, qui envisage décrire, de façon monographique, toutes les fonctions du seul ordre des mots ou du seul accent dynamique de la phrase. Mathesius exige que l'on prenne en considération des phrases formellement diverses, pourvue qu'elles soient réunies par une variation consciente de leur signification. Et c'est précisément dans cette perspective qu'il réunit et retravaille divers articles de sa plume, rédigés au cours de presque trente ans, pour en faire un manuel d'analyse fonctionnelle du langage, intitulé Čeština a obecný jazykozpyt ('Le tchèque et la linguistique générale').

Ici nous suspendons notre exposé sans pourtant l'arrêter ni renvoyer sine die. L'histoire de la perspective communicative de la phrase, c'est-à-dire de l'articulation actuelle de celle-ci, ne peut être saisie que dans dans un contexte englobant toutes les vicissitudes qu'a connues la description de langue au sein du

\footnotetext{
${ }^{11}$ Louis Hjelmslev fait répercuter leur débat structural dans les Acta linguistica II/1940-1941, en publiant un compte-rendu détaillé des deux contributions de NovÁK $(1936,1937)$ réagissant à SKALIČKA (1935) et à MATHESIUS (1936a).

${ }^{12}$ Sa préface est datée «octobre 1941 et novembre 1943 » ce que nous comprenons comme les dates d'une rédaction première et d'une révision ultérieure. Or la censure nazie en ayant définitivement empêché l'impression, le volume a dû attendre jusqu'à 1947. Son auteur est mort le 12 avril 1945.
} 
foyer pragois de structuralisme fonctionnel. À ce but, il faut reconnaitre (et argumenter), pour la période classique du Cercle de Prague :

- le radicalisme de Vilém Mathesius qui refuse la grammaire traditionnelle telle quelle, et au rang d'unité élémentaire de l'analyse linguistique aussi bien que stylistique, élève l'énoncé, tout en cherchant à le doter d'ensembles contrastifs adéquats, au sein desquels se déroule l'établissement différentiel de valeurs linguistiques. Il partageait cette attitude avec Jan Mukařovský qui s'occupait exclusivement d'analyses stylistiques, avec Bohuslav Havránek qui traitait notamment de la langue en tant que supersystème de systèmes dont chacun peut être compris comme une langue fonctionnelle particulière correspondant à un corpus particulier de textes, aussi bien qu'avec Bohumil Trnka, intéressé alors notamment par le dynamisme inhérent des systèmes statiques de langue ;

- la diversité mutuelle des approches structurales de la morphologie traditionnelle adoptées respectivement par Sergej Karcevskij, Nikolaj Trubeckoj et Roman Jakobson. Pour ce dernier, il faut en outre bien distinguer un Jakobson pragois (1920-1939) d'un Jakobson américain (1942-1982) ;

- la modernité du projet de grammaire structurale, bâtie à partir de la notion de sème, envisagée par Vladimír Skalička et Ludovít Novák. Malheureusement, ni l'un ni l'autre - chacun pour des raisons différentes - n'ont pu parachever leur projet en appareil descriptif englobant tout le système de langue ;

- le rôle symbolique, au sein de la communauté scientifique, de deux syntaxes du tchèque moderne élaborées, l'une par František Trávníček, l'autre par Vladimír Šmilauer, deux personnages dont les rapports avec le Cercle de Prague étaient bien délicats.

Les points cités ci-haut concernent la période classique du foyer pragois, lorsque le Cercle exerçait ses activités ouvertement (1926-1948). Viennent ensuite, avec certaines zones de transition, les périodes discrète (1952-1969) et dispersée (1971-1989), pendant lesquelles le Cercle ne pouvait guère exercer ses activités et officiellement n'existait même pas. C'est au cours de cette longue époque-là que se sont constituées les diverses écoles pragoises de perspective fonctionnelle, c'est-à-dire articulation actuelle de la phrase. Leur diversité ne résulte pas d'une ramification successive d'un projet de recherche commun. Elle est due à la transmission discrète, qui comme le temps empirait progressivement, a fini par devenir quasi clandestine, de la tradition fonctionnelle-structurale du foyer: bien que grand nombre des membres du premier Cercle pussent continuer dans leur carrière universitaire et de recherche, ils ne constituaient plus un milieu qui se fréquente, qui discute, dispute, débat; un jeune philologue de talent pouvait être choisi par un «ancien» du Cercle, initié et engagé dans un sillon particulier de travail philologique, sans jamais échanger véritablement avec son collègue, initié par un autre « ancien » et engagé dans un sillon voisin, voire parallèle.

Le contexte des recherches linguistiques de l'époque est marqué par la rédaction de deux grammaires du tchèque contemporain, l'une « modérée », de référence dans l'enseignement secondaire aussi bien qu'universitaire (par Bohuslav Havránek et Alois Jedlička), l'autre exhaustive et systématisante, récusée pourtant dès sa parution comme de «la-science-pour-la-science » (Karel Horálek, Miloš 
Dokulil, František Daneš, Miroslav Komárek, avec un large réseau de collaborateurs $)^{13}$; ces deux n'épuisent pourtant pas, à leur époque, la description scientifique du tchèque. Sur ce fond, apparemment neutre, mais au fait profondément imprégné de la tradition fonctionnaliste du foyer pragois, on peut repérer plusieurs écoles structuralistes autonomes :

- Bohumil Trnka (1895-1984), un des fondateurs du premier Cercle, achève après la guerre, dans une solitude complète, une description fonctionnelle de langue qui, mettant en valeur toute l'expérience du structuralisme classique aussi bien que contemporaine (dans son exile intérieur, Trnka était parfaitement au courant des événements mondiaux), représente la constitution de l'énoncé (y compris son articulation actuelle) à travers plusieurs paliers de complexité. Or restée sans continuation, sa théorie est tombée, hélas ! dans un oubli injuste mais total.

- M. Jan Šabršula (1918), s'inspirant du débat structuraliste de l'après-guerre (notamment dans la lignée Hjelmslev - Coseriu - romanistes européens), développe une description sémiotique du langage, complexe et très originale. L'articulation actuelle de l'énoncé (sic !), strictement binaire (thème : : rhème), y est traitée comme résultant de l'opposition des sèmes isotopants et non isotopants. Aussi dans le cas de l'œuvre de M. Šabršula, la continuation est peu assurée.

- M. František Daneš (1919) choisit Mathesius pour son maître spirituel direct et choisit l'articulation actuelle de la phrase pour un principe fondamental de la syntaxe textuelle qu'il cherche à établir. M. Daneš, tout comme Mathesius (§ 3), travaille consciemment dans les deux sens, dans le linguistique (des textes au système de langue) et dans le stylistique (du système de langue aux textes). Or pour le côté linguistique, M. Daneš adopte un cadre descriptif que nous qualifions de cognitiviste avant la lettre. Son approche de l'articulation actuelle de la phrase résonne dans des théories et analyses stylistiques devenues depuis de référence (Karel Hausenblas, Miroslav Červenka), ce qui lui assure une certaine postériorité, quoique limitée plutôt aux spécialistes du tchèque et des langues slaves.

- Jan Firbas (1921-2000), disciple de Josef Vachek, disciple de Mathesius, remplace le terme d'articulation actuelle de la phrase par celui de perspective fonctionnelle de la phrase $(\S 1)$. Il travaille dans le sens stylistique sans songer à donner un appareil descriptif particulier du système de langue. Son orientation exclusive lui permet de passer de l'articulation mathesiussienne originellement bipartite à toute une gamme de «contributions communicatives ». En outre, Firbas développe la caractérologie mathesiussienne des langues en comparant les stratégies spécifiques déployées par diverses langues pour servir les mêmes buts fonctionnels. De référence notamment dans les milieux anglicistes, l'approche firbassienne est de loin la mieux connue, ce qui ne garantit pourtant pas qu'elle restera activement pratiquée.

\footnotetext{
${ }^{13}$ Jan Petr, rédacteur en chef des trois volumes, qui parce que toujours le premier nommé et imprimé séparément des autres, est, dans les bibliographies, souvent indiqué comme l'auteur principal, était à l'époque un vizir du parti communiste préposé à la rédaction. Les hautes qualités de l'œuvre ne lui sont point redevables.
} 
- M. Petr Sgall (1926), disciple de Vladimír Skalička, disciple de Mathesius, comprend la division actuelle de la phrase comme une couche autonome de la signification de la phrase au sein du système abstrait de langue, ce qui le distingue considérablement des précédents. Deux structures phrastiques différenciées uniquement par la délimitation de la frontière séparant le thème et le rhème, sont par conséquent deux phrases différentes, disposées à servir de support pour deux énoncés différents, non échangeables dans une situation textuelle particulière sans changer ou sans dévier le sens global résultant. ${ }^{14}$

- Oldřich Leška (1927-1997) élabore une description structuraliste de langue remarquable notamment par son entrelacement des dimensions synchronique et diachronique de la langue. Lui aussi considère la division actuelle de la phrase comme une couche autonome de la structure de signification. Cependant, son attention est orientée vers d'autres buts.

Voilà quelques points à développer dans un traité ultérieur...

\section{BIBLIOGRAPHIE}

La bibliographie structurée de la période classique du foyer pragois fera l'objet d'une publication particulière de l'auteur. Ici nous ne donnons qu'une liste partielle des publications collectives principales. Ensuite viennent les références des ouvrages mentionnés dans les $\S \S 1$ à 4 . Par contre, ce qui est dit au sujet des périodes postclassiques du foyer pragois de structuralisme fonctionnel (§5), n'est qu'un canevas d'un futur article, et restera sans référence : choisir, pour chacun des scientifiques ci-mentionnés, deux ou trois ouvrages « symboliques » serait trop osé si fait de façon aléatoire, et nécessiterait tout un exposé, si appuyé sur des motifs explicites.

FOYER PRAGOIS (période classique, publications collectives principales)

TCLP - Travaux du Cercle linguistique de Prague.

TCLP 1. Mélanges linguistiques dédiés au Premier congrès des philologues slaves. 1929.

TCLP 2. Roman Jakobson: Remarques sur l'évolution phonologique du russe comparée à celle des autres langues slaves. 1929.

TCLP 3. Bohumil Trnka: On the syntax of the English verb from Caxton to Dryden. 1930.

TCLP 4. Réunion phonologique internationale tenue à Prague (18-21/XII 1930). 1931.

TCLP 5. Description phonologique du russe moderne. II. Nikolaj Sergeevič Trubeckoj (Trubetzkoy): «Das morphonologische System der russischen Sprache ». 1934.

\footnotetext{
${ }^{14}$ Une illustration de cette approche est offerte par l'auteur (2012), lui-même disciple de M. Sgall.
} 
TCLP 6. Études dédiées au Quatrième congrès de linguistes. 1936

TCLP 7. Nikolaj Sergeevič Trubeckoj (Trubetzkoy): Grundzüge der Phonologie. 1939.

TCLP 8. Études phonologiques dédiées à la mémoire de M.le Prince N.S.Trubetzkoy. 1939.

SaS - Slovo a slovesnost. Časopis Pražského lingvistického kroužku [Le verbe et la création verbale. Revue du Cercle linguistique de Prague], volumes I/1935 jusqu'à IX/1943, X/1947-1948, XI/1948-1949.

CHARISTERIA GVILELMO MATHESIO QVINQVAGENARIO A DISCIPVLIS ET CIRCVLI LINGVISTICI PRAGENSIS SODALIBVS OBLATA. Sumptibus «Pražský linguistický kroužek » (Cercle linguistique de Prague), Pragae, 1932.

Spisovná čeština a jazyková kultura [Le standard littéraire du tchèque et la cultivation de la langue] (ediderunt Bohuslav Havránek et Miloš Weingart). Melantrich, Praha, 1932.

Torso a tajemství Máchova díla. Sborník pojednání Pražského linguistického kroužku [Le fragment et le mystère de l'œuvre de Karel Hynek Mácha. Recueil de travaux du Cercle linguistique de Prague] (edidit Jan Mukařovský). Fr.Borový, Praha, 1938.

Čtení o jazyce a poesii [1] [Lectures sur la langue et la poésie] (ediderunt Bohuslav Havránek et Jan Mukařovský). Družstevní práce, Praha, 1942.

A Prague school reader in linguistics (composuit et edidit Josef Vachek). Indiana university studies in the history and theory of linguistics. Indiana university press, Bloomington, 1964.

\section{RÉFÉRENCES (ouvrages mentionnés)}

Albano Leoni, Federico (2009), Dei suoni e dei sensi. Il volto fonico delle parole, Il Mulino, Bologna.

ARTYMOVYČ, Agenor (1932a), «Pysana mova» [La langue écrite], Zbirnyk Ukrajinśkoho vysokoho pedagogičnoho instytuta v Prazi [Travaux de l'École normale supérieure ukrainienne à Prague] II, p. 1-8.

ARTYMOVYČ, Agenor (1932b), «Fremdwort und Schrift», in : CHARISTERIA..., p. 114-118.

DOVER, Kenneth James (1960), Greek Word Order, Oxford.

FIRBAS, Jan (1957), «Some thoughts on the function of word order in Old English and Modern English», in: Sborník prací filosofické fakulty brněnské university A5, p. 72-100, Brno.

HAVRÁNEK, Bohuslav (1928, 1937), Genera verbi ve slovanských jazycích I, II [La voix verbale dans les langues slaves I, II], Královská česká společnost nauk, Praha. 
HoSkOVEC, Tomáš (2011), Synchronie et diachronie au centre et à la périphérie du foyer pragois de structuralisme fonctionnel, Cahiers Ferdinand de Saussure 64, p. 49-72.

HoskoveC, Tomáš (2012), La linguistique textuelle et le programme de philologie englobante, Verbum XXXII/2010 [sic !], nº 2, p. 193-218, Presses universitaires de Nancy.

JAKOBSON, Roman (1931), Die Betonung und ihre Rolle in der Wort- und Syntagmaphonologie, In TCLP 4, p. 164-182.- Reimpressum in Roman Jakobson, Selected Writings I, p. 117-136.

KARCEVSKIJ, Serge (1931), Sur la phonologie de la phrase, In TCLP 4, p. 188227. - Reimpressum in Serge Karcevski [sic !], Inédits et introuvables, Textes rassemblés et établis par Irina et Gilles Fougeron, p. 87-124. Peeters, Leuven/Louvaine.

MATHESIUS, Vilém (1907-1908), Tainova kritika Shakespeara [La critique de Shakespeare par Hippolyte Taine], in : Věstník České akademie císaře Františka Josefa pro vědy, slovesnost a umění 16/1907, p. 339-354, p. 533-539; 17/1908, p. 227-239. Praha.

MATHESIUS, Vilém (1907-1909), Studie k dějinám anglického slovosledu [Études sur l'évolution de l'ordre des mots en anglais], in : Věstník České akademie císaře Františka Josefa pro vědy, slovesnost a umění 16/1907, p. 261-275; $17 / 1908$, p. $195-216$, p. $299-311$; 18/1909, p. $1-12$; 19/1910, p. $247-257$, p. 258-262, Praha.

MATHESIUS, Vilém (1911), O potenciálnosti jevů jazykových [Sur la potentialité des phénomènes linguistiques], in : Věstník Královské české společnosti nauk 1911-1912, Třída filosoficko-historicko-jazykozpytná 2, p. 1-24, Praha.

MATHESIUS, Vilém (1912), O aposici v moderní angličtině [Sur l'apposition en anglais moderne], Sborník filologický III, p. 240-251. — Retractatum ut «Podstata aposice a její druhy » [La nature de l'apposition et ses genres] in Mathesius 1947, p. 302-318.

MATHESIUS, Vilém (1923), Několik slov o podstatě věty [A propos de la nature de la phrase], Časopis pro moderní filologii X, 1, p. 1-6.

MATHESIUS, Vilém (1924), Několik poznámek o funkci podmětu v moderní angličtině [Quelques remarques sur la fonction du sujet en englais moderne], Časopis pro moderní filologii X, 3/4, p. 244-248. — Retractatum ut «O funkci podmětu » [Sur la fonction du sujet] in Mathesius 1947, p. 277-285.

MATHESIUS, Vilém (1926), Přívlastkové ten, $t a$, to v hovorové češtině [Le pronom démonstratif en tchèque parlé], Naše řeč X, 2, p. 39-41. - Reimpressum in Mathesius 1947, p. 185-189.

MATHESIUS, Vilém (1928) [paru 1930], On linguistic characterology with illustrations from modern English, In Actes du Premier congrès international de linguistes à la Haye du 10-15 avril 1928, p. 56-63, A. W. Sijthoff, Leiden. - Reimpressum in A Prague school reader..., p. 59-67.

MATHESIUS, Vilém (1929a), Zur Satzperspektive im modernen Englisch, Archiv für das Studium der neueren Sprachen und Literaturen, Jahrgang 84, Band 155, p. 202-210, G.Westermann, Braunschweig - Berlin - Hamburg. 
MATHESIUS, Vilém (1929b), Funkční linguistika [La linguistique fonctionnelle], in: Sborník prednášek pronesených na Prvém sjezdu československých profesoru filosofie, filologie a historie v Praze 3-7.dubna 1929, p. 27-39. «Funktionale Linguistik», in: Stilistik und Soziolinguistik, Beiträge der Prager Schule zur strukturellen Sprachbetrachtung und Spracherziehung, List Verlag, Berlin 1971.

MATHESIUS, Vilém (1930), K pořádku slov v hovorové češtině [A propos de

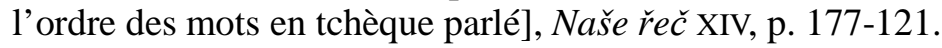

MATHESIUS, Vilém (1931), K dynamické linii české věty [A propos de la ligne dynamique de la phrase tchèque], Časopis pro moderní filologii XVII, p. 71-81.

MATHESIUS, Vilém (1936a), Pokus o teorii strukturální mluvnice [Essai d'une théorie de la grammaire structurale], $S a S$ II, p. 47-54.

MATHESIUS, Vilém (1936b), Deset let pražského lingvistického kroužku [Dix années du Cercle linguistique de Prague], SaS II, p. 137-145. - "Ten years of the Prague linguistic circle", in: Josef Vachek, The linguistic school of Prague. An introduction to its theory and practice, p. 137-151, Indiana university press, Bloomington 1966.

MATHESIUS, Vilém (1937a), Mluvní takt a některé problémy příbuzné [La mesure de la parole et quelques problèmes apparentés], SaS III, p. 193-199.

MATHESIUS, Vilém (1937b), K teorii větné intonace [Au sujet de la théorie d'intonation de phrase], $S a S$ III, p. 248-249.

MATHESIUS, Vilém (1938), Zesílení a zdůraznění jako jevy jazykové [Le renforçage et l'emphase en tant que phénomènes linguistiques], $S a S$ IV, p. 193202.

MATHESIUS, Vilém (1939a), Verstärkung und Emphase, in: Mélanges de linguistique offerts à Charles Bally, p. 407-414, Georg et $c^{\text {ie }}$, Genève. Reimpressum in A Prague school reader..., p. 426-432, Abrégé allemand de l'article tchèque 1938.

MATHESIUS, Vilém (1939b), O tak zvaném aktuálním členění věty [Sur la soidisant articulation actuelle de la phrase], $S a S$ v, p. 171-174.

MATHESIUS, Vilém (1940), Vyjadřování pasivní perspektivy v české větě [L'expression de la perspective passive dans la phrase tchèque], Listy filologické XLVII, 3-4 (Festschrift Oldřichu Hujerovi), p. 307-311.

MATHESIUS, Vilém (1941a), Rozpor mezi aktuálním členěním souvětí a jeho organickou stavbou [Divergence entre l'articulation actuelle et la construction organique de la période], $S a S$ VII, p. 37-40.

MATHESIUS, Vilém (1941b), Základní funkce pořádku slov v češtině [Les fonctions principales de l'ordre des mots en tchèque], SaS VII, p. 169-180.

MATHESIUS, Vilém (1942), Řeč a sloh [Le langage et le style], in : Čtení o jazyce..., p. 10-100.

MATHESIUS, Vilém (1943), Jazykozpytné poznámky k řečnické výstavbě souvislého výkladu [Remarques de linguiste sur la structuration rhétorique de l'exposé], $S a S$ IX, p. 114-129.

MATHESIUS, Vilém (1947), Čeština a obecný jazykozpyt [La langue tchèque et la linguistique générale], Melantrich, Praha, Choix d'études préparé par l'auteur en 1941 et revu en 1943, empêché d'imprimer par l'occupant nazi. 
Mathesius, Vilém (2009), Paměti a jiné rukopisy [Mémoires et d'autres manuscrits], Karolinum, Praha.

MuKAŘOVSKÝ, Jan (1928), Máchưv Máj [Le poème Máj de Karel Hynek Mácha] (avec un résumé en français), Karlova universita, Praha.

MUKAŘOVSKÝ, Jan (1929), Rapports de la ligne phonique avec l'ordre des mots dans les vers tchèques, in : TCLP 1 , p. 121-145.

MUKAŘOVSKÝ, Jan (1931), La phonologie et la poétique, in : TCLP 4, p. 278-288.

MUKAŘOVSKÝ, Jan (1938), Genetika smyslu v Máchově díle [La genèse du sens dans l'œuvre de Mácha], in : Torso a tajemství..., p. 13-110.

NovÁK, Ludovít (1936), Základná jednotka gramatického systému a jazyková typológia [L'unité fondamentale du système grammatical et la typologie linguistique], Sborník Matice slovenskej pre jazykospyt, literárnu historiu, dejepis a národopis, XIV/1936.

NovÁK, L’udovít (1937), K základným otázkám štrukturálnej jazykovedy [Au sujet de quelques questions cruciales de la linguistique structurale], Sborník Matice slovenskej pre jazykospyt, literárnu historiu, dejepis a národopis, XV/1937.

SKALIČKA, Vladimír (1935), Zur ungarischen Grammatik, Universita Karlova, Praha. - Reimpressum in Vladimír Skalička, Typologische Studien (edidit Paul Hartmann), p. 59-125, Vieweg, Braunschweig - Wiesbaden 1979.

SKALIČKA, Vladimír (1936), La fonction de l'ordre des éléments linguistiques, in : TCLP 6, p. 129-133.

SKALIČKA, Vladimír (1937), Promluva jako linguistický pojem [L'énonciation comme notion de la linguistique], SaS III, p. 163-166.

VACHEK, Josef (1939), Zum Problem der geschriebenen Sprache, TCLP 8, p. 94104. - Reimpressum in A Prague school reader..., p. 453-460.

VACHEK, Josef (1942), Psaný jazyk a pravopis [La langue écrite et l'orthographe], in : Čtení o jazyce..., p. 229-306.

VACHEK, Josef (1999), Prolegomena k dějinám Pražské školy jazykovědné, H\&H, Jinočany. - "Prolegomena to the history of the Prague School of Linguistics", in: Prague Linguistic Circle Papers (Travaux du Cercle linguistique de Prague, nouvelle série) 4, p. 3-81, John Benjamins, Amsterdam - Philadelphia.

TRÁVNíČEK, František (1937), Základy československého slovosledu [Éléments de l'ordre des mots tchécoslovaque], SaS III, p. 78-86.

TRÁVNíČEK, František (1939), Slovosled při důrazu [L'ordre des mots sous l'emphase], SaS v, p. 131-144.

TRNKA, Bohumil (1932), Some thoughts on structural morphology, in : CHARISTERIA..., p. 57-61. 